\title{
Correction to: Basic Conditions of Early Mathematics Education-a Comparison between Germany, Taiwan and Switzerland
}

\author{
Hedwig Gasteiger ${ }^{1}$ (D) Esther Brunner ${ }^{2} \cdot$ Ching-Shu Chen $^{3}$ \\ Published online: 27 January 2020 \\ (C) Ministry of Science and Technology, Taiwan 2020
}

\section{Correction to: International Journal of Science and Mathematics Education https://doi.org/10.1007/s10763-019-10044-x}

The original version of this article unfortunately contains incorrect formatting of section 2 sub-headings, section 5 and Tables 2 to 5 with typing errors in Tables 4 and 5 .

The corrected section 2 sub-headings and section 5 should be

\subsection{Relevance of Socio-economic Status}

\subsection{Influence of Linguistic Aspects in Different Languages}

2.3 Influence of Culture

\subsection{Influence of Instruction and Curriculum}

\section{Results}




\section{The corrected formating and typing errors of Tables 2 to 5 should be}

Table 2 Comparison of the educational system

\begin{tabular}{|c|c|c|c|}
\hline & Taiwan & Germany & Switzerland \\
\hline Age of kindergarteners & $3-6$ years & $3-6.5$ years & $4.5-6.5$ years \\
\hline Compulsory education & Not compulsory & Not compulsory & Compulsory \\
\hline Kindergarten fees & 5-6 years none & Yes & None \\
\hline Part of the school system & Yes & No & Yes \\
\hline $\begin{array}{l}\text { Weekly time children } \\
\text { spend in kindergarten }\end{array}$ & $35 \mathrm{~h}$ & $\begin{array}{l}\text { Depends on } \\
\text { parents booking }\end{array}$ & $\begin{array}{l}\text { 4-5 years: } 20 \mathrm{~h} \\
\text { 5-6 years: } 24 \mathrm{~h}\end{array}$ \\
\hline Responsibility & Public, private & $\begin{array}{l}\text { Many private } \\
\quad \text { organizations, public }\end{array}$ & $\begin{array}{l}\text { Public, very few } \\
\text { private organizations }\end{array}$ \\
\hline
\end{tabular}

Table 3 Pre-service education

\begin{tabular}{llll}
\hline & Taiwan & Germany & Switzerland \\
\hline Kind of education & University studies & $\begin{array}{l}\text { Vocational school in 2 stages: } \\
\text { 1: Social assistant, } \\
\text { 2: Educator }\end{array}$ & University studies \\
Required certification & High school exam & $\begin{array}{l}\text { 1: Middle school } \\
\text { 2: Social assistant }\end{array}$ & $\begin{array}{c}\text { General qualification for } \\
\text { university entrance }\end{array}$ \\
Mathermatics education & Yes & No & Yes \\
Graduation & University certificate & State certified educator & BA in pre-primary \\
& & & education \\
\hline
\end{tabular}

Table 4 Legal requirements

\begin{tabular}{lllc}
\hline & Taiwan & Germany & Switzerland \\
\hline $\begin{array}{l}\text { Curriculum } \\
\begin{array}{c}\text { Mathematical } \\
\text { content }\end{array}\end{array}$ & $\begin{array}{l}\text { Inner-mathematical character } \\
\text { age 3-4: } \text { - numbers }\end{array}$ & $\begin{array}{l}\text { Literacy-based } \\
\text { Very diverse, depends on } \\
\text { the different curricula } \\
\text { ine 5-6: geometry, numbers, } \\
\text { measurement }\end{array}$ & $\begin{array}{c}\text { Counting, numbers, } \\
\text { comparison, time, } \\
\text { geometry }\end{array}$ \\
Benchmarks & Yes & No & Yes \\
\hline
\end{tabular}


Table 5 Working conditions

\begin{tabular}{|c|c|c|c|}
\hline & Taiwan & Germany & Switzerland \\
\hline $\begin{array}{l}\text { Salary compared to } \\
\text { primary school teachers }\end{array}$ & Comparable & Less & Less \\
\hline $\begin{array}{l}\text { Students/kindergarten } \\
\text { teacher relation }\end{array}$ & $\approx 15 / 1$ & $\begin{array}{l}\approx 25 / 2 \text { (educator }+ \text { social } \\
\quad \text { assistant }\end{array}$ & $\approx 20 / 1$ \\
\hline $\begin{array}{l}\text { Additional human } \\
\text { resources }\end{array}$ & $\begin{array}{l}\text { Special needs teacher } \\
\text { (some hours weekly) }\end{array}$ & $\begin{array}{l}\text { Trainee, volunteer, } \\
\text { if required: trained } \\
\text { assistant for language } \\
\text { promotion }\end{array}$ & $\begin{array}{l}\text { Special needs teacher } \\
\text { for kindergarten } \\
\text { and school }\end{array}$ \\
\hline
\end{tabular}

\section{The original article has been corrected.}

Publisher's Note Springer Nature remains neutral with regard to jurisdictional claims in published maps and institutional affiliations. 